\title{
Statins and postoperative delirium
}

\author{
Edward R. Marcantonio MD SM
}

$\infty$ See related research paper by Redelmeier and colleagues, page 645

$\mathrm{D}$ elirium (acute confusional state) may be the most common postoperative complication in elderly people. It is associated with substantial morbidity, mortality and cost. ${ }^{1}$ Two decades ago, the prevalent belief was that it was normal for older patients to be a "bit confused" after surgery. Now, delirium is recognized as an important complication that should be prevented, or promptly recognized, evaluated and treated. Using current evidence, clinicians can identify patients at risk for postoperative delirium (Box 1), ${ }^{2}$ address modifiable risk factors ${ }^{3-5}$ and implement intervention strategies to prevent or reduce delirium. ${ }^{6,7}$ Effective strategies include geriatric consultation, which reduces the incidence of delirium by over one-third after hip-fracture repair, ${ }^{6}$ and prophylactic low-dose haloperidol, which reduces the severity and duration of delirium after high-risk hip surgery.

The development and dissemination of techniques to validly and reliably diagnose delirium, such as the Confusion Assessment Method, ${ }^{8}$ have been central to progress in delirium research. This method assesses 4 key features of delirium, the diagnosis of which requires the presence an acute change in mental status with a fluctuating course and inattention, with either disorganized thinking or an altered level of consciousness. The Confusion Assessment Method diagnostic algorithm, which is used with a short, standardized mental status examination, can be completed in 15-20 minutes by trained, nonclinician interviewers. This algorithm has 90\%-95\% sensitivity and specificity relative to a "goldstandard" assessment by a geriatrician or geriatric psychiatrist. ${ }^{8}$ The Confusion Assessment Method has become widely adopted in delirium research and clinical practice.

In this issue of CMAJ, Redelmeier and colleagues report a significant association between the perioperative use of statins and postoperative delirium (odds ratio 1.30, 95\% confidence interval 1.15-1.47). ${ }^{9}$ They performed a large-scale pharmacoepidemiology study using the Canadian Institutes for Health Information database. They identified 284158 patients aged 65 years or older admitted to the hospital for elective surgery in Ontario from 1992-2002. Drug exposures to statins and other classes of drugs were identified using a population-wide prescription database. The authors identified cases of delirium using hospital discharge abstracts (International Classification of Disease, ninth revision [ICD-9] codes 293.0-293.9).

A general rule of epidemiology is that a study is only as good as its principal outcome measure. The outcome measure for delirium used by Redelmeier and colleagues has serious

\section{Key points}

- Delirium is a common, morbid and costly complication of elective surgery in elderly patients.

- Delirium is associated with a number of reversible factors. Correcting these factors may reduce the incidence of delirium.

- Use of statins during the perioperative period may increase the risk of postoperative delirium by about $30 \%$.

- The methods used in the study by Redelmeier and colleagues underestimate the true incidence of delirium.

- The association between the use of statins and delirium requires confirmation using other methods.

limitations. The authors cite a study that coding of delirium in the discharge abstract has a sensitivity of $35 \%$ and a specificity of $98 \%{ }^{10}$ compared with the gold standard of medical record review. However, studies that have compared medical record reviews to direct patient interviews have demonstrated that only $10 \%-50 \%$ of delirium cases are documented in the medical record. ${ }^{11}$ Given the loss of sensitivity from both medical record reviews and discharge abstract coding, it is probable that less than $10 \%$ of delirium cases were identified in this study. This explains the discrepancy between the $1.1 \%$ delirium rate reported and the $15 \%-25 \%$ delirium rate after elective surgery reported using validated methods for primary data collection. ${ }^{2}$

The authors present a series of analyses to strengthen their argument that the use of statins increases the risk of postoperative delirium. First, they correctly argue that coding of delirium in the discharge abstract, although not sensitive, is probably highly specific; that is, there are few "false positives." They present 2 approaches commonly used in pharmacoepidemiology: a dose-response analysis showing that the risk of delirium increases with increasing doses of statin, and a drugwithdrawal analysis showing that patients previously prescribed a statin but no longer taking it at the time of surgery were not at increased risk.

Atherosclerosis has emerged as a major risk factor for delirium, ${ }^{12}$ and statins are used to treat patients with atherosclerosis. The authors address this potential "confounding by indication" by use of stratification (exclusion of patients with major vascular disorders), statistical adjustment and propensity score matching. In each case, delirium remained associ-

Dr. Marcantonio is with the Division of General Medicine and Primary Care, Beth Israel Deaconess Medical Center, Harvard Medical School, Boston, Mass. 


\section{Box 1: Risk factors for postoperative delirium}

Predisposing factors (present before surgery, most not modifiable)

- Aged $\geq 70$ years

- Cognitive impairment (dementia)

- Functional impairment

- High medical comorbidity

- Markedly abnormal laboratory values

- Alcohol abuse

- Sensory impairments (vision, hearing loss)

Precipitating factors (occurring during or after surgery, many modifiable)

- High-risk surgery (cardiac surgery, noncardiac thoracic surgery, vascular surgery, aortic aneurysm repair, hip-fracture repair)

- Postoperative benzodiazepines

- Postoperative meperidine analgesia

- Poorly controlled postoperative pain

- Low postoperative hematocrit

- Postoperative complications

ated with the use of statins, though the adjustor variables also came from the discharge abstracts and are limited. The authors examined a long list of other cardiac medications, vascular medications and miscellaneous medications, and they found no other associations with delirium. A much shorter list of neuropsychiatric medications shows the expected associations with delirium. All of these analyses strengthen the authors' argument.

The assessment of causality requires a biological mechanism. Beyond their direct effect on lipids, statins have direct effects on microvascular tone, which may be mediated through the expression of endothelial nitric oxide synthase. The authors suggest that this direct effect, which may be beneficial in the prevention of myocardial infarction and stroke, may shunt blood away from neurons postoperatively. This shunting of blood may lead to ischemia, which in turn may predispose patients to delirium. Inadequate cerebral oxygenation is one of the current models of pathophysiology for delirium. Thus, the authors' theory is plausible.

In the face of these strengths and weaknesses, how does one judge the value of this article and its implications for clinical practice? Clinical investigations are conducted on a continuum: some studies are better designed to generate hypotheses and others are better designed to test them. The study by Redelmeier and colleagues represents a valuable addition to the literature. It proposes and eloquently defends an association between statins and postoperative delirium. However, the low detection of the primary outcome increases the risk of un- measured confounding and bias that cannot be fully addressed by even the excellent methods employed. For example, if delirium were under-coded by as little as $3 \%$ among patients not using statins compared with those using statins, this could account for the authors' findings. Therefore, I believe the association between statins and delirium requires confirmation in studies using rigorous, state-of-the-art methods developed for the assessment and diagnosis of delirium.

What is the clinician to do right now? Unlike the authors, I believe it is premature to recommend stopping the use of statins in elderly surgical patients. The methodology used in this study is simply too limited to compel practice change. Moreover, we do not know the effects of stopping statins on other perioperative outcomes. Finally, in our increasingly fragmented health care system, there is the risk that the statins would not be restarted after surgery, leading to long-term harm to patients. Despite these caveats, I believe that the study by Redelmeier and colleagues presents a compelling argument to further explore the association between statins and postoperative delirium. Given the increasing age of the surgical population and the prevalence, morbidity, and cost of postoperative delirium, even a modest risk reduction would translate to substantial benefit for patients and the health care system.

Competing interests: None declared.

\section{REFERENCES}

1. Inouye SK. Current concepts: delirium in older persons. N Engl J Med 2006;354: 1157-65.

2. Marcantonio ER, Goldman L, Mangione CM, et al. A clinical prediction rule for delirium after elective non-cardiac surgery. JAMA 1994;271:134-9.

3. Marcantonio ER, Juarez G, Goldman L, et al. The relationship of postoperative delirium with psychoactive medications. JAMA 1994;272:1518-22.

4. Lynch EP, Lazor MA, Gellis JE, et al. The impact of postoperative pain on the development of postoperative delirium. Anesth Analg 1998;86:781-5.

5. Marcantonio ER, Goldman L, Orav EJ, et al. The association of intraoperative factors with the development of postoperative delirium. Am J Med 1998;105:380-4.

6. Marcantonio ER, Flacker JM, Wright JR, et al. Reducing delirium after hip fracture: a randomized trial. J Am Geriatr Soc 2001;49:516-22.

7. Kalisvaart KJ, de Jonghe JFM, Bogaards MJ, et al. Haloperidol prophylaxis for elderly hip surgery patients at risk for delirium: a randomized placebo-controlled trial. J Am Geriatr Soc 2005;53:1658-66.

8. Inouye SK, van Dyck $\mathrm{CH}$, Alessi CA, et al. Clarifying confusion: the Confusion Assessment Method. A new method for detection of delirium. Ann Intern Med 1990;113:941-8.

9. Redelmeier DA, Thiruchelvam D, Daneman N. Delirium after elective surgery among elderly patients taking statins. CMAJ. 2008;179:645-52.

10. Romano PS, Chan BK, Schembri ME, et al. Can administrative data be used to compare postoperative complication rates across hospitals? Med Care 2002;40:856-67.

11. Johnson JC, Kerse NM, Gottlieb G, et al. Prospective versus retrospective methods of identifying patients with delirium. J Am Geriatr Soc 1992;40:316-9.

12. Rudolph JL, Jones RN, Rasmussen LS, et al. Independent vascular and cognitive risk factors for postoperative delirium. Am J Med 2007;120:807-13.

Correspondence to: Dr. Edward R. Marcantonio, Division of General Medicine and Primary Care, Beth Israel Deaconess Medical Center, 1309 Beacon St. \#218, Brookline MA 02446, USA; fax 617 754-1440 